\title{
Correction to: The androgen receptor/filamin A complex as a target in prostate cancer microenvironment
}

Marzia Di Donato, Alice Zamagni, Giovanni Galasso, Erika Di Zazzo (D, Pia Giovannelli, Maria Vittoria Barone, Michele Zanoni, Roberta Gunelli, Matteo Costantini, Ferdinando Auricchio, Antimo Migliaccio (D, Anna Tesei (i) and Gabriella Castoria (1D

Correction to: Cell Death \& Disease https://doi.org/10.1038/s41419-021-03402-7 published online 26 January 2021

The original version of this article unfortunately contained a mistake. Roberta Gunelli is affiliated with the Department of Urology, Morgagni Pierantoni Hospital,
Forli, Italy and Matteo Costantini is affiliated with Pathology Unit, Morgagni Pierantoni Hospital, Forlì, Italy. The authors apologize for the mistake. The original article has been corrected.

Published online: 15 March 2021 